\title{
Evaluación de competencias digitales de estudiantes de la Universidad Católica de Santo Toribio de Mogrovejo (Perú)
}

Recibido: 6 de octubre de 2019 / Revisado: 9 de octubre de 2019

Aceptado: 4 de noviembre de 2019 / Publicado: 20 de diciembre de 2019

ROCÍO DEL PILAR RUMICHE CHÁVARRY

Departamento Humanidades, Universidad Católica de Santo Toribio de Mogrovejo, Perú rrumiche@usat.Edu.Pe

GERARDO RAÚL CHINGA CHINGUEL

Departamento Humanidades, Universidad Católica de Santo Toribio de Mogrovejo, Perú gchunga@usat.edu.pe

http://dx.doi.org/10.24310/IJNE2.2.2019.7452

\section{RESUMEN}

La investigación se ha realizado en la Universidad Católica de Santo Toribio de Mogrovejo en Chiclayo (Perú). La alfabetización digital y la competencia digital son claves hoy para todos los ciudadanos (para su desarrollo personal, su integración social, acceso a la cultura y el conocimiento, poder comunicarse y recibir información, herramienta laboral y lúdica, ...) siendo una herramienta indispensable para los estudiantes universitarios, tanto para el aprendizaje como para el desarrollo de la competencia investigativa. El objetivo, de la investigación, es conocer en qué medida la dimensión informacional, que es parte la competencia digital, está adquirida por los estudiantes de esa Universidad. La metodología usada ha sido tipo encuesta, a través de la aplicación de un cuestionario.

Los resultados de la investigación es que, a pesar de la importancia, del acceso a la información de calidad para los estudiantes universitarios, el nivel de la mayoría de los estudiantes de la muestra, en cuanto a dominio de la dimensión informacional, está en proceso de lograrlo y una octava parte de ellos, ya ha conseguido dominar la dimensión informacional.

Palabras Clave: Alfabetización digital, competencias, educación superior.

\section{ABSTRACT}

Evaluation of digital competencies of students of the Catholic University of Santo Toribio de Mogrovejo (Perú)

The research has been carried out at the Catholic University of Santo Toribio de Mogrovejo in Chiclayo (Peru). Digital literacy and digital competence are key today for all citizens (for their personal development, social integration, access to culture and knowledge, to be able to communicate and receive information, work and recreational tools, ...) being an indispensable tool for University students, both for learning and for the development of research competence. The objective, of the research, is to know to what extent the informational dimension, which is part of the digital competence, is acquired by the students of that University. The methodology used has been a survey type, through the application of a questionnaire.

The results of the research is that, despite the importance of access to quality information for university students, the level of the majority of the students in the sample, in terms of mastery of the informational dimension, is in process to achieve it and an eighth of them, has already managed to dominate the informational dimension.

Keywords: Digital literacy, competencies, higher education. 


\section{Introduction}

\subsection{Problemática}

Una de las principales causas y efectos de la globalización se debe el fuerte avance que se ha tenido en el desarrollo de tecnologías relacionadas con la comunicación y la información. La aparición de equipos y programas, cada vez más baratos y fáciles de usar, han permitido un enorme avance en la conectividad de los seres humanos. Eso ha convertido literalmente al planeta Tierra, en lo que se considera una Aldea Global, donde forma instantánea nos podemos comunicar con seres humanos residentes en distintos lugares con un coste económico pequeño.

Igualmente, la información fluye de forma rápida, y los ciudadanos han pasado de ser receptores pasivos de información a generar y transmitir información, con equipos tan simples como los celulares o teléfonos móviles. Ello ha conllevado una forma más democrática de acceso a la información y a las posibilidades de comunicar. No obstante, este transitar no está exento de peligros y dificultades. Es por ello, que, desde organismos internacionales, se aboga por promover la alfabetización digital como una competencia básica de todos los ciudadanos, y, por supuesto, conseguir que esa alfabetización digital tenga un elevado nivel en los estudiantes universitarios. En la actualidad parecería que, dada el elevado número de equipos tecnológicos y aplicaciones, que usan los estudiantes universitarios, la alfabetización digital y la competencia tecnológica debe estar adquirida por los mismos, independientemente de la Universidad y del país en que residan. Pero ¿realmente los estudiantes universitarios, de poblaciones medias y alejadas de las grandes urbes, como son los de Chiclayo, están alfabetizados digitalmente?

En esta investigación nos planteamos, como objetivo, conocer en qué medida la competencia digital está adquirida por los estudiantes de la Universidad Católica de Santo Toribio de Mogrovejo, y dado que la competencia digital es muy amplia nos centraremos en la dimensión informacional.

\subsection{Fundamentación teórica}

Comenzaremos abordando, en primer lugar, el concepto de competencia, para posteriormente sumergirnos en la competencia digital y alfabetización tecnológica.

El Proyecto Desing and Selectión of Competencies (DeSeCo, 2005) promovido por la OCDE, considera que las competencias son más que destrezas y conocimientos, puesto que incorpora habilidades psicosociales para atender demandas complejas, que surgen en un entorno particular. DeSeCo incorpora competencias claves que deben adquirir todos los seres humanos y entre ellas destacan usar herramientas interactivas. 
En un amplio análisis del concepto de competencias, realizado por López (2016), al responder ¿qué son? las aglutina en habilidades cognitivas que hacen alusión al conocimiento conceptual y al saber conocer; capacidades, habilidades prácticas y destrezas relacionadas con el conocimiento procedimental y el saber hacer; y finalmente motivaciones, emociones y valores vinculadas con el conocimiento actitudinal y el saber ser.

En el contexto iberoamericano, a pesar de que existen críticas al modelo de competencias, el informe realizado por la Organización para la Cooperación y el Desarrollo Económico (OCDE), el Banco de Desarrollo de América Latina (CAF) y la Comisión Económica para América Latina y el Caribe de Naciones Unidad (CEPAL), en el 2015, sugiere a los gobiernos emprender reformas, en la educación, las competencias y la innovación, para conseguir un mayor crecimiento con equidad y un fortalecimiento de la clase media. El informe destaca que hay una mayor demanda de competencias altas y de las denominadas competencias de carácter general o blandas, que permiten una mejor adaptación de las personas a entornos cambiantes. Igualmente, insisten que los sistemas educativos deben permitir que la población desarrolle las competencias necesarias para su integración, en todos los ámbitos de la vida, promoviendo de esta forma una mayor cohesión social y un crecimiento inclusivo en la Región. Las competencias junto a la innovación permitirán el incremento de la productividad laboral, crear empleos de mayor calidad y disminuir la lacra de la economía informal (OCDE; CAF y CEPAL, 2015).

Según Vilches y Gil (2012) "se persigue que todos los profesionales que terminen sus estudios en la universidad hayan adquirido competencias básicas para tomar decisiones y realizar sus actividades desde la perspectiva de la sostenibilidad" (p. 30).

Por su parte la UNESCO (2015) reconoce la transformación que están produciendo las tecnologías digitales, en la vida de lo ciudadanos (desde el trabajo al ocio) en las comunicaciones, en las relaciones internacionales, y en las relaciones sociales. Pero muestra su preocupación por temas relacionados con la privacidad de las personas y los ciberdelitos. Esta preocupación por los malos usos y riesgos, así como la necesidad de adquirir la competencia digital, les hace reclamar al profesorado, una mejor preparación de las generaciones de nativos digitales, para enfrentar las dimensiones éticas y sociales de las tecnologías actuales y de las que puedan desarrollarse en un futuro.

En el informe Horizon (2015) dentro de los desafíos solucionables, en la Educación Superior los expertos incluyen la mejora de la alfabetización digital, aunque asumen que hay que precisar mejor a qué se refiere dicha alfabetización. Resaltando, por ejemplo, diferencias importantes en lo que los estudiantes universitarios consideran plagio, en función de su Universidad o país. Los expertos señalan como una herramienta muy útil la integración de las tecnologías en las prácticas pedagógicas innovadoras. No obstante, entre los que consideran desafíos muy difíciles (incluyen aquellos que son difíciles incluso de definir y mucho más de solucionar) la implementación de modelos de educación en competencia. Se 
considera que los programas de estudios universitarios centrados en el dominio de competencias, claramente definidas, va en aumento, ofreciendo una mayor flexibilidad y una evaluación sumativa. Pero los expertos, críticos, afirman que se deben examinar estos enfoques para asegurar que sean eficaces y que proporcionen beneficios a largo plazo.

Para precisar mejor a qué nos referimos con alfabetización digital y competencia digital acudimos a algunos autores.

La UNESCO (2011) nos presenta dos ámbitos distintos la alfabetización informacional y la mediática. La primera, se concreta en que las personas puedan definir y articular sus necesidades de información; localizar y evaluar información; organizar la información, hacer un uso ético de la información, comunicar información y uso del conocimiento de las TIC para procesar información. Sobre la alfabetización mediática la centra en entender el papel de los medios de comunicación en las sociedades democráticas; evaluar de forma crítica los contenidos y las funciones de los medios y producir, como usuarios, contenidos. En nuestra investigación nos vamos a centrar especialmente en la alfabetización informacional, aunque recogemos también la alfabetización mediática. La integración de ambas es lo que denomina Alfabetización Mediática e Informacional (AMI).

San Nicolás, Fariña y Area (2012, p. 12), recogen las cinco dimensiones vinculadas al nuevo concepto de alfabetización digital dentro del marco de las competencias digitales y las concretan en:

a. Dimensión instrumental: saber usar la tecnología. Es necesario adquirir las habilidades instrumentales para hacer un uso efectivo de los recursos disponibles.

b. Dimensión cognitiva-intelectual: saber transformar la información en conocimiento. Saber plantear problemas, analizar e interpretar con significado la información.

c. Dimensión socio-comunicativa: saber expresar y comunicarse con otros en la red: saber crear documentos textuales e hipertextuales y audiovisuales para participar en redes sociales.

d. Dimensión axiológica: actuar con responsabilidad y valores. Desarrollo de actitudes, valores y prácticas éticas y democráticas en la red.

e. Dimensión emocional: construir una identidad de participación en la web equilibrada emocionalmente.

Durán, Gutiérrez y Prendes (2016, p. 99) definen la competencia digital como “el conjunto de valores, creencias, conocimientos, capacidades y actitudes en aspectos tecnológicos, informacionales y comunicativos que nos lleva a una alfabetización múltiple compleja”.

Para Marín (2013) vincula la adquisición de la competencia digital, de los estudiantes universitarios, con la investigación y el trabajo académico. Ello implicará conocimientos sobre el uso de hardware y software, la creación de una actitud crítica, y la habilidad para difundir y publicar la información. 
El logro por parte de los alumnos de la competencia digital supone el desarrollo de dos aspectos básicos (competencias básicas). Habilidades para buscar, obtener, procesar y comunicar la información y transformarla en conocimiento. Incluye aspectos diferentes que van desde el acceso y selección de la información hasta el uso y la transmisión de ésta en distinntos soportes, incluyendo la utilización de las tecnologías de la información y la comunicación como un elemento esencial para informarse y comunicarse.” (Marín, 2013, p. 90).

Dado la complejidad y polisemia de la competencia digital, estamos de acuerdo con Cabero y Marín (2014) que consideran que ésta engloba a otras competencias y subcompetencias. En la investigación que se describe en este artículo nos centraremos en una subcompetencia.

En Perú según la investigación realizada por Gallardo, Marqués y Gisbert (2011) los formadores de formadores y los docentes que brindan la capacitación dentro del marco del Programa Nacional de Formación y Capacitación Permanente (PRONAFCAP) no cuentas con competencias TIC. Ello dificulta evidentemente, la formación del profesorado y que los estudiantes de nivel inicial, primaria y secundaria puedan adquirir los niveles básicos, intermedio o nivel avanzado de competencia digital.

Por otro lado, no podemos negar el impacto y la hibridación que se ha producido entre las TIC y la economía en el proceso de globalización. Es por ello, que la competencia digital adquiera aún mayor relevancia, para las personas y la sociedad en general. Castell e Himanen (2016) hablan de desarrollo informacional, en un entorno de sociedad global, que genera una nueva forma de organización sociotecno-económica que denominan informacionismo, cuya característica "es la amplia utilización de información digital y tecnología de comunicación... lo que permite la difusión de organización en red en todos los dominios de la vida económica y social” (p. 30).

Pasamos a continuación, tras el acercamiento teórico, a concretar el contenido de la investigación realizada.

\section{Metodología e instrumento}

\subsection{Metodología}

El contexto de la investigación en la Universidad Católica de Santo Toribio de Mogrovejo (USAT), está radicada en la ciudad de Chiclayo, en el norte de Perú. Es una institución de educación superior privada, a la que asisten estudiantes, en general de clase media y media alta. La Universidad tiene aproximadamente unos 8.030 estudiantes y ofrece una amplia gama de titulaciones de grado y posgrado, en las ramas de las Ciencias Empresariales, Derecho, Humanidades, Ingenierías y Medicina. 
Los estudiantes que cursan esos estudios tienen, en general, acceso a diversas tecnologías de la información y de la comunicación (TIC) y se presupone que tienen adquiridas por su edad, recursos y contexto socio-cultural las competencias digitales, que le permiten poder optimizar el uso de las TIC para su vida personal, social y para los estudios. Este artículo, responde a una investigación más amplia, que se especificará de forma breve, en el instrumento.

La metodología utilizada es tipo encuesta, a través de un instrumento adaptado a la realidad sociocultural de los estudiantes de la USAT.

\subsection{Instrumento}

El material o instrumento que se ha utilizado, para obtener la información, ha sido un cuestionario sobre competencia digital que se elaboró en la Universidad de la Sabana de Colombia. El cuestionario consta de 25 ítems o preguntas. Las preguntas están relacionadas con distintos supuestos relacionados con el desarrollo de un proyecto de aula. El proyecto consiste en que los estudiantes propongan soluciones y medidas preventivas sobre problemas medio ambientales relacionados con la contaminación del aíre, del agua, y la inadecuada gestión de los residuos urbanos. Las preguntas son cerradas, y a partir de supuestos o situaciones, que se les presenta a los estudiantes, tendrán que elegir una o varias respuestas que son las correctas.

Los 25 ítems están agrupados en relación a cuatro dimensiones. La dimensión comunicativa tiene 5 ítems; la dimensión informacional tiene 8 ítems; la dimensión tecnológica tiene 4 ítems y la dimensión ciudadanía digital tiene 8 ítems.

El instrumento sufrió un proceso de revisión y adaptación, realizado por un equipo de 5 investigadores, atendiendo a la cultura (significados culturales, comportamientos, normas, hábitos, valores) y las barreras de lenguaje. Igualmente, se hizo una adecuación a la forma de puntuar en Perú.

En el proceso de validación del instrumento, éste fue enviado a 6 profesionales que indicaron que el cuestionario sí evalúa las 4 dimensiones: informacional, comunicativa, tecnológica y ciudadanía digital.

Antes de la aplicación, a una muestra más amplia, se realizó un estudio piloto, con estudiantes de la Universidad Santo Toribio, que estudiaban en el segundo semestre del año 2016. La prueba se realizó con una muestra de 104 estudiantes, la selección fue no probabilística, porque se realizó entre el alumnado que recibía clases de los profesores que formaron el equipo de investigación. El instrumento fue puesto a disposición de los estudiantes a través de la plataforma Limesurvey. 
En la validación y confiabilidad del instrumento, se hizo la validación del constructo mediante el análisis factorial y la validación de contenido mediante el juicio de expertos. El alfa de cronbach del instrumento fue 0,732 (véase tabla 1). La medida obtenida del coeficiente de confiabilidad es excelentemente confiable, según Herrera $(1998,101)$, quien plantea los siguientes valores: 0.53 a menos $=$ Confiabilidad nula; 0.54 a $0.59=$ Confiabilidad baja $; 0.60$ a $0.65=$ Confiable $; 0.66$ a $0.71=$ Muy Confiable; 0.72 a $0.99=$ Excelente confiabilidad; y $1=$ Confiabilidad perfecta .

Tabla 1. Cuadro de Confiabilidad: Estadígrafo Alfa de Cronbach

\begin{tabular}{|c|c|c|}
\hline Alfa de Cronbach & $\begin{array}{c}\text { Alfa de Cronbach basada en elementos } \\
\text { estandarizados }\end{array}$ & N de elementos \\
\hline 0.732 & 0.776 & 25 \\
\hline
\end{tabular}

\section{Resultados}

El cuestionario se pasó en el año 2018, y la muestra estuvo constituido por 1.038 estudiantes. A continuación, en la tabla, se recoge la distribución de la muestra dividida en función de la licenciatura (14 estudios) que cursaban.

Tabla 2. Estudios de licenciaturas

\begin{tabular}{|c|c|c|c|c|}
\hline $\begin{array}{l}\text { D2. ESCUELA } \\
\text { PROFESIONAL }\end{array}$ & FREQUENCY & PERCENT & $\begin{array}{c}\text { VALID } \\
\text { PERCENT }\end{array}$ & $\begin{array}{l}\text { CUMULATIVE } \\
\text { PERCENT }\end{array}$ \\
\hline Administración & 183 & 17.6 & 17.6 & 17.6 \\
\hline $\begin{array}{c}\text { Administración Hotelera } \\
\text { y de Servicios }\end{array}$ & 44 & 4.2 & 4.2 & 21.9 \\
\hline Arquitectura & 115 & 11.1 & 11.1 & 32.9 \\
\hline Comunicación & 60 & 5.8 & 5.8 & 38.7 \\
\hline Contabilidad & 40 & 3.9 & 3.9 & 42.6 \\
\hline Economía & 56 & 5.4 & 5.4 & 48.0 \\
\hline Educación Inicial & 37 & 3.6 & 3.6 & 51.5 \\
\hline $\begin{array}{c}\text { Ingeniería Ambiental } \\
\text { y Civil }\end{array}$ & 144 & 13.9 & 13.9 & 65.4 \\
\hline Ingeniería Industrial & 136 & 13.1 & 13.1 & 78.5 \\
\hline $\begin{array}{c}\text { Ingeniería Mecánica } \\
\text { Eléctrica }\end{array}$ & 37 & 3.6 & 3.6 & 82.1 \\
\hline
\end{tabular}




\begin{tabular}{|c|c|c|c|c|}
\hline $\begin{array}{c}\text { D2. ESCUELA } \\
\text { PROFESIONAL }\end{array}$ & FREQUENCY & PERCENT & $\begin{array}{c}\text { VALID } \\
\text { PERCENT }\end{array}$ & $\begin{array}{c}\text { CUMULATIVE } \\
\text { PERCENT }\end{array}$ \\
\hline $\begin{array}{c}\text { Ingeniería de Sistemas } \\
\text { y Computación }\end{array}$ & 91 & 8.8 & 8.8 & 90.8 \\
\hline Odontología & 17 & 1.6 & 1.6 & 92.5 \\
\hline Psicología Humana & 78 & 7.5 & 7.5 & 100.0 \\
\hline Total & $\mathbf{1 0 3 8}$ & $\mathbf{1 0 0 . 0}$ & $\mathbf{1 0 0 . 0}$ & \\
\hline
\end{tabular}

Como hemos señalado, anteriormente, el cuestionario se centra en cuatro dimensiones la informacional, la comunicativa, la ciudadanía digital y la tecnológica. En este artículo nos vamos a centrar en la informacional, que está medida a través de 7 ítems.

Para solucionar el problema ambiental que se le plantea, a los estudiantes, relacionado con la contaminación del agua, del aire y mal manejo de la basura, y se les comenta que deben buscar soluciones mediadas por TIC (como por ejemplo divulgación, educación, ...) Los estudiantes deberán buscar información y en el cuestionario les da cinco opciones que pueden hacer en Internet. Las opciones y la distribución de las respuestas, queda reflejada en la siguiente tabla.

Tabla 3. Opciones temáticas de búsqueda de información para documentar el proyecto

\begin{tabular}{|c|c|c|c|c|}
\hline $\begin{array}{c}\text { TEMÁTICAS DE } \\
\text { BÚSQUEDA DE TEMÁTICAS }\end{array}$ & FREQUENCY & PERCENT & $\begin{array}{c}\text { VALID } \\
\text { PERCEN }\end{array}$ & $\begin{array}{c}\text { CUMULATIVE } \\
\text { PERCENT }\end{array}$ \\
\hline $\begin{array}{c}\text { Consecuencias de la crisis } \\
\text { ambiental en municipios } \\
\text { zona centro de XXX }\end{array}$ & 47 & 4.5 & 4.5 & 4.5 \\
\hline $\begin{array}{c}\text { Impacto en el ecosistema } \\
\text { de la zona de influencia }\end{array}$ & 46 & 4.4 & 4.4 & 9.0 \\
\hline $\begin{array}{c}\text { Promoción con TIC de protocolos } \\
\text { para preservar } \\
\text { el medio ambiente de XXX }\end{array}$ & 152 & 14.6 & 14.6 & 23.6 \\
\hline $\begin{array}{c}\text { Protocolos y tratados internacionales } \\
\text { para prevenir la crisis ambiental } \\
\text { en la zona de influencia }\end{array}$ & 223 & 21.5 & 21.5 & 45.1 \\
\hline $\begin{array}{c}\text { Soluciones TIC para enfrentar } \\
\text { la crisis ambiental }\end{array}$ & 570 & 54.9 & 54.9 & 100.0 \\
\hline Total & $\mathbf{1 0 3 8}$ & $\mathbf{1 0 0 . 0}$ & $\mathbf{1 0 0 . 0}$ & \\
\hline
\end{tabular}


Los estudiantes podían elegir una sola opción. De las cinco opciones la de "soluciones TIC para enfrentar la crisis ambiental" se considera como la correcta o más pertinente, ya que vincula las dos variables esenciales que debe tener el proyecto: atender problemas ambientales y usar las TIC como herramientas.

Los resultados nos muestran que más del 50\% de los estudiantes han elegido la opción correcta. No obstante, también resalta que el $45.1 \%$ haya seleccionado opciones que no son las más pertinentes; y dentro de ellas destaca que los mayores porcentajes lo tengas los protocolos y tratados internacionales, lo que sugiere una confianza de los estudiantes en estos tipos de acuerdos.

En el ítem 2, se les solicitaba, a los estudiantes, que seleccionaran herramientas para la búsqueda de información, y podían elegir más de una (entre las cinco opciones que se la daban). Dos de ellas Blogger y Monografias.com se consideraban como no pertinentes y las otras tres como adecuadas. Los Blogger son elegidos por $11.7 \%$ de la muestra, y las Mongrafías.com por el 27.6\%, es decir, más de la cuarta parte de los estudiantes. La distribución de las respuestas entre las opciones pertinentes destaca con el mayor porcentaje Google con un porcentaje del $80.8 \%$ y como segunda opción Chrome con un porcentaje del $55.1 \%$. Una diferencia del $25.7 \%$ a favor de Google que muestra la fuerte hegemonía de este buscador. Por último, la opción de Sciencie Direct tiene también un alto porcentaje, el 42.9\%, indicando que es una herramienta también muy utilizada por los estudiantes.

En el ítem 3 los estudiantes debían seleccionar dos opciones. Las opciones eran: a) búsqueda sencilla; b) búsqueda cruzada; c) búsqueda avanzada; d) búsqueda por material; e) búsqueda por fecha. Las opciones que se consideraban más inadecuadas son la búsqueda por material, la búsqueda cruzada y la búsqueda por fecha. El menor porcentaje, de respuesta afirmativas, lo tiene la opción de búsqueda cruzada con un $9 \%$. En el polo opuesto el $78.9 \%$ optan, por la respuesta correcta, búsqueda avanzada. La búsqueda por material es elegida por casi la mitad de los estudiantes, el 48.9\%, y con un porcentaje menor, aunque bastante alto, el $40.8 \%$, está la búsqueda por fecha.

En el ítem 4, se ofrecía, a los estudiantes, cinco combinaciones de palabras, para buscar información relevante para el proyecto. Se introdujeron, entre las palabras claves, nombres de localidades, de la zona de Chiclayo, que son conocidas por los estudiantes, ya que incluso muchos viven en ellas.

Tabla 4. Selección de las palabras claves para la búsqueda de información

\begin{tabular}{|c|c|c|c|c|}
\hline $\begin{array}{c}\text { Seleccione las palabras claves } \\
\text { que considere indispensable para } \\
\text { continuar con la búsqueda } \\
\text { de información: }\end{array}$ & FREQUENCY & PERCENT & $\begin{array}{c}\text { VALID } \\
\text { PERCEN }\end{array}$ & $\begin{array}{c}\text { CUMULATIVE } \\
\text { PERCENT }\end{array}$ \\
\hline $\begin{array}{c}\text { Medio ambiente, aire, agua, Chicla- } \\
\text { yo, Lambayeque, Ferreñafe }\end{array}$ & 67 & 6.5 & 6.5 & 6.5 \\
\hline
\end{tabular}




\begin{tabular}{|c|c|c|c|c|}
\hline $\begin{array}{c}\text { Seleccione las palabras claves } \\
\text { que considere indispensable para } \\
\text { continuar con la búsqueda } \\
\text { de información: }\end{array}$ & FREQUENCY & PERCENT & $\begin{array}{c}\text { VALID } \\
\text { PERCEN }\end{array}$ & $\begin{array}{c}\text { CUMULATIVE } \\
\text { PERCENT }\end{array}$ \\
\hline $\begin{array}{c}\text { Protocolo Kioto, contaminación } \\
\text { tierra, TIC, Chiclayo, manejo de } \\
\text { plástico papel }\end{array}$ & 112 & 10.8 & 10.8 & 17.2 \\
\hline $\begin{array}{c}\text { Solución TIC, contaminación, at- } \\
\text { mósfera, agua }\end{array}$ & 196 & 18.9 & 18.9 & 36.2 \\
\hline $\begin{array}{c}\text { Solución, TIC, contaminación tierra, } \\
\text { medio ambiente }\end{array}$ & 401 & 38.6 & 38.6 & 74.9 \\
\hline $\begin{array}{c}\text { Solución, contaminación, manejo de } \\
\text { residuos, TIC, Lambayeque }\end{array}$ & 261 & 25.1 & 25.1 & 100 \\
\hline Total & $\mathbf{1 0 3 8}$ & $\mathbf{1 0 0 . 0}$ & $\mathbf{1 0 0 . 0}$ & \\
\hline
\end{tabular}

Los estudiantes podían elegir sola una opción como la correcta. La opción idónea, era "Solución TIC, contaminación, atmósfera y agua”. Esta opción generaba algunas dificultades, puesto que, aunque se indicaba que era un proyecto genérico, no ubicado en ninguna localidad, los estudiantes podían inclinarse por incluir en sus búsquedas el nombre de ciudades. Por otro lado, no figuraba literalmente el manejo inadecuado de las basuras, sino que se incluía durante el concepto más amplio de contaminación. Estas dificultades hicieron que solo el 18.9\% de la muestra eligieran lo opción correcta, y que el $42.4 \%$ de los estudiantes se inclinaran por combinaciones de palabras que incluían el nombre de alguna ciudad.

En el ítem 5 se le indicaba, a los estudiantes, que hay palabras claves que se normalizan en función de las áreas de conocimiento para facilitar la búsqueda de información y el trabajo de los especialistas. A continuación, se les preguntaba donde podían encontrar esas palabras claves. En la siguiente tabla se puede apreciar la distribución de las respuestas.

Tabla 5. Dónde buscar palabra claves normalizadas

\begin{tabular}{|c|c|c|c|c|}
\hline $\begin{array}{c}\text { P5. Para usar palabras claves } \\
\text { normalizadas según el área de } \\
\text { conocimiento, se debe utilizar: }\end{array}$ & FREQUENCY & PERCENT & $\begin{array}{c}\text { VALID } \\
\text { PERCEN }\end{array}$ & $\begin{array}{c}\text { CUMULATIVE } \\
\text { PERCENT }\end{array}$ \\
\hline Buscadores & 368 & 35.5 & 35.5 & 35.5 \\
\hline Normalizadores & 101 & 9.7 & 9.7 & 42.2 \\
\hline Sinónimos & 316 & 30.4 & 30.4 & 75.6 \\
\hline Tesauros & 60 & 5.8 & 5.8 & 81.4 \\
\hline Vocabulario & 193 & 18.6 & 18.6 & 100.0 \\
\hline Total & $\mathbf{1 0 3 8}$ & $\mathbf{1 0 0 . 0}$ & $\mathbf{1 0 0 . 0}$ & \\
\hline
\end{tabular}


Entre las cinco opciones la respuesta correcta es la de tesauros. Podemos observar como es la opción elegida por el menor porcentaje de los estudiantes que componen la muestra. Destaca que los mayores porcentajes lo tengan los buscadores y los sinónimos.

En el ítem 6, y continuando con la búsqueda de información para su proyecto y las palabras claves, se les ofrecieron 4 opciones como estrategia para encontrar información confiable, y sólo debían elegir una opción.

La respuesta elegida por el mayor porcentaje de estudiantes fue la de "Solución TIC, medio ambiente, contaminación, basura y, reciclaje y aire" con el 40.1\%. La solución correcta, que incluye todos los elementos claves del proyecto es "Solución TIC, manejo basuras, contaminación agua y contaminación aire" es seleccionada por el $32.9 \%$ de la muestra. La opción más genérica que recoge "Solución TIC, medio ambiente y reciclaje" alcanza el $20,1 \%$ de respuestas de la muestra. Y con el menor porcentaje está "TIC, medio ambiente, contaminación y agua" con un $16.1 \%$ de respuestas.

Por último, en el ítem 7, se coloca a los estudiantes en un supuesto donde la búsqueda con las palabras claves le ha arrojado un resultado de 40 publicaciones. A partir de esa realidad se les pregunta sobre los criterios que elegirían para evaluar la información encontrada. A los alumnos se les dan distintas opciones y se les dice que elijan las tres más importantes.

Las opciones son: a) el idioma en que se encuentra el documento; b) si se identifica claramente al autor (es) del documento; c) si el documento tiene enlaces a otros sitios; d) si los temas de los contenidos corresponden a la información buscada; e) si el documento tiene imágenes referenciadas; f) si el documento tiene referencias bibliográficas confiables; g) si el documento está en formato electrónico.

Las opciones consideradas como más pertinentes, la identificación clara del autor ha sido elegida por el $63.1 \%$ de los estudiantes; si los temas de los contenidos corresponden a la información buscada es seleccionada por el $73.3 \%$ y finalmente "si el documento tiene referencias bibliográficas confiables" es la respuesta del $70.8 \%$.

Respecto a la valoración final de la competencia digital, se la asignó a cada una de las dimensiones un total de 5 puntos. Al ser cuatro dimensiones el máximo de puntos era 20. Como en este artículo solo hemos tenido en cuenta la dimensión informacional, el máximo sería de 5 puntos.

Si nos atenemos a los niveles de logro, de la competencia digital, que hemos considerado, en función de la puntuación, tendremos los siguientes: no logrado, inicial, en proceso, logrado y logrado destacado.

Al valorar las respuestas de los estudiantes nos encontramos que el 60\%, de los mismos, respecto a la dimensión informacional tiene un nivel de en proceso; ninguno de los estudiantes está en el nivel 
máximo más alto de logro destacado, ni de no logrado. El 27\% de los estudiantes está en un nivel inicial y el $12 \%$ ha logrado tener la competencia digital en esta dimensión.

\section{Discusión}

La mayoría de los estudiantes de la Universidad Católica de Santo Toribio de Mogrovejo, respecto a la competencia digital, está en proceso de conseguir el dominio de la dimensión informacional. Siendo preocupante que, una cuarta parte de los estudiantes, esté en un nivel inicial en esta competencia, tan vital para el trabajo académico y para la investigación.

En una investigación con estudiantes de Pedagogía (Flores y Roig, 2016), de la Universidad de Bío-Bío (Chile), realizada por recoge que la mayoría de los estudiantes han adquirido el conocimiento del uso de TIC de forma autodidacta, y que hay deficiencias en el dominio de la competencia TIC, por problemas inherentes a los propios estudiantes a la oferta académica que hace la Facultad de Educación.

En el estudio realizado por Gutiérrez y Cabero (2016) con estudiantes universitarios de Educación, de la Comunidad de Andalucía (España), sobre alfabetización tecnológica, incluyen como categoría de análisis la búsqueda y tratamiento de la información. En esta investigación los estudiantes las puntuaciones vienen oscilando entre la puntuación de 7.95 (sobre 10) en la capacidad de localizar información a través de diferentes fuentes y bases de datos, y el 6.95 de "planificar búsquedas de información para la resolución de problemas". Destacamos también la puntuación de 7.52 para "saber identificar la información relevante adecuando distintas fuentes y su procedencia”.

Al contrastar, los datos de las investigaciones, se observa que hay un menor dominio de la alfabetización tecnológica, en los estudiantes de las dos universidades americanas. No obstante, se evidencia que es necesario que los estudiantes, de las universidades en las que se han producido las investigaciones, tengan un mayor dominio de la competencia digital. Por ello, las universidades y profesores universitarios deberían evaluar, de forma continua, esta competencia y diseñar estrategias pertinentes, para conseguir que, los estudiantes, tengan un adecuado dominio de la competencia digital.

\section{Conclusiones}

Entre las capacidades básicas en una era de sobresaturación de información es discriminar la relevante, analizar, reflexionar, relacionar información y sacar conclusiones, sólo de esta forma los ciudadanos podrán ser activos, participativos y podrán propiciar sus propios procesos de desarrollo personal. 
En nuestra investigación, nos hemos centrado especialmente, en si los estudiantes de la Universidad de Santo Toribio de Mogrovejo, dentro de su competencia digital tienen un dominio de la misma que les permita buscar información relevante, de calidad y de fuentes fiables para poder insumar un proyecto de investigación.

Los datos nos señalan los estudiantes de la USAT, no tienen un dominio óptimo, de la dimensión informacional de la competencia digital,

No se puede considerar que la abundancia de equipos y programas de tecnologías de la información y de la comunicación generan, por si misma, la alfabetización digital y la adquisición de la competencia digital. Es por ello que, es necesario evaluar, de forma sistemática, si efectivamente los estudiantes, y especialmente los universitarios, la han adquirido.

Además, hay que destacar que es necesaria diseñar estrategias, para asegurar que todos los estudiantes consigan un nivel óptimo de dominio, de la alfabetización digital, para poder desarrollar todo su potencial, en su propio proceso de aprendizaje y para tener competencias investigativas. Propiciándole, de este modo, herramientas para que sea responsable de su propio proceso de autoformación, potenciar su socialización y la integración en los distintos entornos laborales, que el futuro le brinde.

\section{Limitaciones del estudio}

El tamaño de la muestra es lo suficientemente amplio para representar a la población. No obstante, las correlaciones que se han detectado son débiles y no permiten llegar a conclusiones definitivas, pero todo apunta a que efectivamente los estudiantes de la Universidad Católica de Santo Toribio de Mogrovejo no tienen un nivel alto de alfabetización tecnológica.

\section{Financiación}

La investigación no ha recibido ninguna financiación, sino que ha surgido por el interés científico de los investigadores.

\section{Prospectiva}

La evaluación continua de las competencias digitales, en sus distintas dimensiones, es una herramienta útil para los estudiantes y para las universidades para poder orientar la toma de decisiones. Es por ello, que esperamos trabajar en la realización de más evaluaciones y en la mejora de las herramientas de evaluación 


\section{Referencias bibliográficas}

Cabero, J. y Marín, V. (2014). Miradas sobre la formación del profesorado en tecnologías de la información y comunicación (TIC).Enl@ce: Revista Venezolana de Información y Conocimiento, 11 (2), 11-24.

Castells, M. e Himanen, P. (2016). Modelos de desarrollo en la era globlal de la información: construcción de un marco analítico. En M. Castells y P. Himanen (coords), Reconceptualización del Desarrollo en la Era Global de la Información (pp. 27-44). Santiago de Chile: Fondo de Cultura Económica.

Durán, M.; Gutiérrez, I. y Prendes, M. P. (2016). Análisis conceptual de modelos de competencia digital del profesorado universitario. Revista Latinoamericana de Tecnología RELATEC, 15 (1), 97-114

Flores, C. y Roig, R. (2016). Percepción de estudiantes de Pedagogía sobre el desarrollo de su competencia digital a lo largo de su proceso formativo. Estudios Pedagógicos, 13 (3), 129-148.

Gallardo, E.E.; Marqués, L. y Gisbert, M. (2011). Propuestas de competencias TIC en el marco del Programa Nacional de Formación y Capacitación Docente (PRONAFCAP). Revista Iberoamericana de Educación, 54 (6), 1-13.

Gutiérrez, J. y Cabero, J. (2016). Estudio de caso sobre la autopercepción de la competencia digital del estudiante universitario de las titulaciones de grado de Educación Infantil y Primaria. Profesorado. Revista de curriculum y formación del profesorado, 20 (2), 180-199.

Johnson, L.; Adams, S.; Estrada, V. \& Freeman, A. (2015). NMC Horizon Report: Edición Educación Superior. Austin, Texas: The New Media Consortium. Recuperado de http://www. funciva.org/wp-content/uploads/2016/11/Horizon-Repor-2015.pdf

López, E. (2016). En torno al concepto de competencia: un análisis de fuentes. Profesorado Revista de currículum y formación del profesorado, 20 (1), 311-322.

Marín, V. (2013). Competencias digitales los alumnos la organización de un PLE y su utilización en la formación. En M. C. Sonseca (coord.), Los entornos personales de aprendizaje (pp. 84-98). Caracas: Universidad Metropolitana.

OCDE, CAF y CEPAL (2015). Perspectivas económicas de América Latina 2015. Educación, com- 
petencias e innovación para el desarrollo. Recuperado de https://repositorio.cepal.org/ bitstream/handle/11362/37445/S1420759 es.pdf

UNESCO (2011). Alfabetización mediática e informacional. Currículum para profesores. París: UNESCO. Recuperado de https://informate.campusfad.org/recursos/documentos/ AMI Unesco.pdf

UNESCO (2015). Replantear la educación ¿Hacia un bien común mundial? París: UNESCO. Recuperado de https://unesdoc.unesco.org/ark:/48223/pf0000232697

Vilches, A. y Gil, D. (2012). La educación para la sostenibilidad en la Universidad: el reto de la formación del profesorado. Profesorado. Revista de Currículum y Formación del Profesorado, 16 (2), 25-43.

San Nicolás, M. B.; Fariña, E. y Area, M. (2012). Competencias digitales del profesorado y alumnado en el desarrollo de la docencia virtual. Revista Historia de la Educación Latinoamericana, 19 (14), 227-245. 
